\title{
Lower hybrid oscillating two-stream instability in a plasma with magnetic shear
}

\author{
By J. M. WERSINGER† \\ Ecole Polytechnique Fédérale de Lausanne, Switzerland
}

\section{A. H. KRITZ}

Ecole Polytechnique Fédérale de Lausanne, Switzerland, and Plasma Physics Laboratory, Princeton University, Princeton, N.J. 08540

\section{AND F. TROYON}

Ecole Polytechnique Fédérale de Lausanne, Switzerland

(Received 16 May 1978 and in revised form 6 October 1978)

Magnetic shear is found to have a strong effect on the propagation characteristics of the lower hybrid parametric daughter waves but no significant effect on the pump wave. The analysis of the OTS instability shows that the convective damping introduced by magnetic shear acts on a distance $L \cdot \simeq H\left(m_{e} / m_{i}\right)^{\frac{1}{2}}$, where $H$ is the magnetic shear scale length. There are two regimes for the convective damping, depending on the wavelength of the parametric daughter waves. For small wavelengths the growth rates are linear functions of $(k L)^{-1}$. For large wavelengths the growth rates are exponentially decreasing functions of $(k L)^{-1}$.

\section{Introduction}

In recent years there has been a strong interest in lower hybrid heating. Initially, the idea was that the incident wave energy would be absorbed by linear mechanisms (Landau or collisional damping) near the lower hybrid resonance layer (Stix 1965). However, there has been experimental evidence (Hooke \& Bernabei 1972; Chu, Bernabei \& Motley 1973; Chang \& Porkolab 1973, 1974) and theoretical predictions (Kindel, Okuda \& Dawson 1972) that nonlinear processes, i.e. parametric instabilities, may become effective in absorbing the incident wave energy (Chen \& Berger 1977; Rogister \& Hasselberg 1976). A variety of decay channels have been investigated both for a dipole pump (Kindel et al. 1972; Porkolab 1974a; Berger \& Perkins 1976) and for a pump with a finite wavelength (Ott 1975). However, it is not straightforward to extrapolate results for an infinite homogeneous plasma to results for a realistic fusion-type plasma.

The model is improved by taking into account finite pump extent (Kuo \& Chen 1976; Berger et al. 1977; Porkolab 1977) as well as density and magnetic

$\dagger$ Present address: School of Electrical Engineering, Cornell University, Ithaca, NY 14853.

$\ddagger$ Permanent address: Hunter College/CUNY, New York, NY 10021. 
field inhomogeneities (Ott 1975; Porkolab 1974b; Porkolab 1977). It is well known that, in an inhomogeneous plasma, the rays of the parametric daughter waves bend, thus allowing their energy to escape from the region of strong coupling (Perkins \& Flick 1971; Rosenbluth 1972). This phenomenon is called convective damping since, like a damping process, it reduces the growth rates and increases the threshold of the instability. In this paper we focus on magnetic shear. Preliminary results have been presented previously in abbreviated form (Wersinger, Kritz \& Troyon 1976). Here, we derive and extend these results to a new regime (as discussed below). As a model we consider a cold plasma with constant density but varying magnetic field described in slab geometry. In typical tokamaks the density profile is sharp at the edge and almost flat in the core of the plasma. Consequently, our results are more likely to apply to the behaviour of lower hybrid waves in the central part of tokamaks.

A measure of the effect of shear on lower hybrid wave propagation is the distance required for the wave rays to rotate by an angle of $\frac{1}{4} \pi$. It emerges that, for the parametric daughter waves, this distance is of the order of $H\left(m_{e} / m_{i}\right)^{\frac{1}{2}}$ where $H$ is the shearing length (the distance over which the direction of the magnetic field linesrotate by an angle of $\frac{1}{4} \pi$ ). For the launched pump wave, namely, either the fast wave or the slow wave, the distance for the rays to turn is of the order of $H\left(m_{i} / m_{e}\right)^{\frac{1}{2}}$. Thus, shear strongly affects those lower hybrid waves which may be destabilized by the pump. More precisely, the parametric daughter waves may encounter turning points near the core of the plasma and experience increased Landau damping. On the other hand, magnetic shear does not introduce a new turning point for the pump wave, and does not influence Landau damping.

The incident pump wave has a frequency $\omega_{0}$ which is close to the local lower hybrid resonance frequency, $\omega_{L H}$, in the centre of the tokamak. In this regime, the so-called dipole approximation predicts that the dominant parametric processes are the non-resonant (or quasi-mode) decay instability and the OTSI (oscillating two-stream instability) (Porkolab 1974a). It has been shown, in an inhomogeneous plasma, that the non-resonant decay instability is a convective instability (Ott 1975; Porkolab 1974b). This result holds in the case of a sheared plasma since the convective nature is an intrinsic property of non-resonant decay (Ott 1975). In this paper we focus on the effect that shear has on OTSI. Both experimental (Chang \& Porkolab 1974) and theoretical (Kindel et al. 1972) work have shown the effectiveness of OTSI as a heating mechanism in the lower hybrid frequency range. It has been pointed out that the OTS instability is a feature of the dipole pump approximation and that finite values of the pump wavenumber $k_{0}$ introduce a finite real low frequency $\omega$ (Fried et al. 1976). We now determine how small $k_{0}$ has to be in order for the OTSI model to be valid. Let $\omega_{L H}$ and $\gamma_{L H}$ designate the real and imaginary parts of the lower hybrid decay wave frequency. Clearly, $\omega<\gamma_{L H}$ is the most stringent condition. The OTS instability occurs for

$$
\left|\omega_{0}-\omega_{L H}\right| \approx \gamma_{L H}
$$

so that we obtain from the work of Fried etal. the condition $k_{0}<\gamma_{L H} / v_{g}$, where $v_{g}$ is the group velocity of the decay waves. This inequality is best satisfied for the fast 
wave heating scheme and/or for short wavelength decay waves. Our analysis applies particularly well to the fast wave scheme, since this wave does not propagate in resonance cones. Thus, the convection of decay wave energy out of the strongly unstable zone is essentially in the radial direction and is due to magnetic shear.

We find that in a plasma with magnetic shear the OTSI is an absolute instability, the growth rate being an eigenvalue. The convective damping introduced by shear acts on the effective scale length $L=H\left(m_{e} / m_{i}\right)^{\frac{1}{2}}$ thus strongly reducing the growth rates. However, unlike previous results on absolute instabilities (Perkins \& Flick 1971), we find a regime where the growth rates are no longer reduced proportional to $(k L)^{-1}$ (Wersinger et al. 1976), but instead are exponentially decreasing functions of $(k L)^{-1}$. Thus, for small values of $k L$ the growth rate reduction is weaker than one might have expected. Moreover, the exponential reduction of growth rates leads to an accumulation of the eigenvalues. These results are the consequence of the nature of lower hybrid waves whose group velocity decreases for increasing wavenumber $k$.

In the next section, we study the effect of shear on lower hybrid wave propagation (both for the launched slow wave and the decay-type waves). It is easy to verify that shear has no significant effect on the fast wave. The dispersion relation is obtained in slab geometry using fluid equations. The limitations due to Landau damping are then introduced phenomenologically. In $\S 3$ we derive the two secondorder coupled differential equations which describe lower hybrid waves under OTSI conditions. In $\$ 4$, the coupled equations are solved in two limits. For wave propagation regions shorter than $L$, we use a Fourier transform method and for longer regions of propagation we use the WKB method. The results are discussed in $\S 5$. In this work we use MKS units.

\section{Effect of magnetic shear on wave propagation}

As a model, we consider a cold homogeneous plasma subject to a sheared magnetic field described in slab geometry by

$$
\mathbf{B}_{0}=B_{0}\left(\hat{z}+\frac{x}{H} \hat{y}\right),
$$

where $B_{0}$ is the constant axial magnetic field, and $H$ is the shearing length. The $x, y$ and $z$ variables correspond to the radial, poloidal and toroidal directions of a torus.

For our model, the dispersion relation of the lower hybrid waves can be written

$$
k_{x}^{2}(x)=\frac{\omega_{L H}^{2}}{\omega^{2}-\omega_{L H}^{2}} \frac{m_{i}}{m_{e}} k_{\|}^{2}(x)-k^{2}
$$

where

$$
\begin{gathered}
\omega_{L H}^{2}=\frac{\omega_{p i}^{2}}{1+\omega_{p e}^{2} / \omega_{c e}^{2}}, \quad k=|\mathbf{k}|=\left(k_{y}^{2}+k_{z}^{2}\right)^{\frac{1}{2}}, \\
k_{\|}^{2}(x)=\left(k_{z}+\frac{x}{H} k_{y}\right)^{2} /\left(1+\frac{x^{2}}{H^{2}}\right) .
\end{gathered}
$$

For sufficiently large $k_{\|}$, the lower hybrid waves are strongly Landau damped (not included in (2), the cold plasma dispersion relation); whereas, for sufficiently 
small $k_{1}, k_{x}^{2}$ given by (2) becomes negative, and the waves are evanescent. In lower hybrid heating one deals essentially with two types of waves. These are the launched waves with $k_{y} \simeq 0$, and the waves that are parametrically driven unstable with $k_{y} \gg k_{z}$. We shall show that the parametric daughter waves may

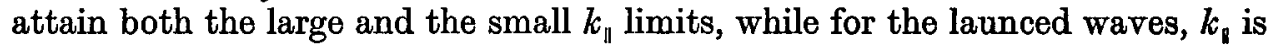
nearly constant.

With the aid of (2), we reduce the two conditions for weak Landau damping, i.e. $k_{\sharp}^{2} V_{t e}^{2} \ll \omega^{2}$ and $\left(k_{x}^{2}+k^{2}\right) V_{t i}^{2} \ll \omega^{2}$ to a single condition on $k_{n}(x)$ :

$$
\frac{k_{0}^{2}(x) V_{t e}^{2}}{\omega^{2}} \ll \min \left[1, \frac{T_{e}}{T_{i}} \frac{\omega^{2}-\omega_{L H}^{2}}{\omega_{L H}^{2}}\right],
$$

where the first and second terms in the bracket are associated with electron and ion Landau damping, respectively. Here, $\nabla_{t e}\left(V_{t i}\right)$ is the electron (ion) thermal speed. For sufficiently large $k_{\|}$, the condition in (4) is violated and the wave is absorbed. In the opposite limit, i.e. small $k_{\rrbracket}$, it follows from (2) that a turning point occurs at a position $x_{t}$ defined by

$$
k_{\sharp}^{2}\left(x_{t}\right)=\frac{m_{e}}{m_{i}} \frac{\omega^{2}-\omega_{L H}^{2}}{\omega_{L H}^{2}} k^{2} .
$$

Thus for lower hybrid waves to propagate, $k_{\|}$must satisfy the condition in (4) and also be larger than the value of $k_{\|}$given by (5).

For the launched lower hybrid waves $\left(k_{y}=0\right),(3 b)$ reduces to

$$
k_{\| 1}^{2}(x)=k_{z}^{2}\left(1+x^{2} / H^{2}\right)^{-1},
$$

which is nearly constant in the region of interest. As a result, Landau damping of the wave spectrum is unaffected by magnetic shear. Moreover, for the launched slow waves there is no turning point inside the plasma as a result of shear. We recall that density variations are neglected here. It is readily seen from (5) that $\left|x_{t}\right| \gg H$ for the launched slow waves which have $k_{\text {凡 }}$ given by (6).

Next we consider the parametric decay waves. These waves have $k_{y} \gg k_{z}$ so that the variation of $k_{\|}$with $x$ is significant. Electron Landau damping completely absorbs the wave when $k_{\|} V_{t e} \simeq \omega$, i.e. when $x=x_{L}$ where

$$
x_{L}=H\left(\frac{\omega}{k_{y} V_{t e}}-\frac{k_{z}}{k_{y}}\right) \text {. }
$$

Clearly, for large $k_{y}, x_{L}$ may be located inside the plasma. In addition, paramotrically excited waves may reach the turning point $x_{t}$, defined in (5), at the location

where

$$
\begin{gathered}
x_{t} \simeq x_{0}+L\left(1+k_{z}^{2} / k_{y}^{2}\right)^{\frac{1}{2}}, \\
x_{0}=-\frac{k_{z}}{k_{y}} H, \quad L=H\left(\frac{m_{e}}{m_{i}} \frac{\omega^{2}-\omega_{L H}^{2}}{\omega_{L H}^{2}}\right)^{\frac{1}{2}} .
\end{gathered}
$$

We observe that $\left|x_{t}\right| \ll H$. As a result, lower hybrid parametric decay-type waves propagate in a region along $x$ limited by

$$
x_{0}+L \lesssim x<x_{0}+H \omega / k_{v} V_{t e}
$$

The expression for $k_{x}^{2}$ in (2) can be written

$$
k_{x}^{2} \simeq k_{y}^{2}\left[\left(x-x_{0}\right)^{2} / L^{2}-1\right]-k_{z}^{2}
$$




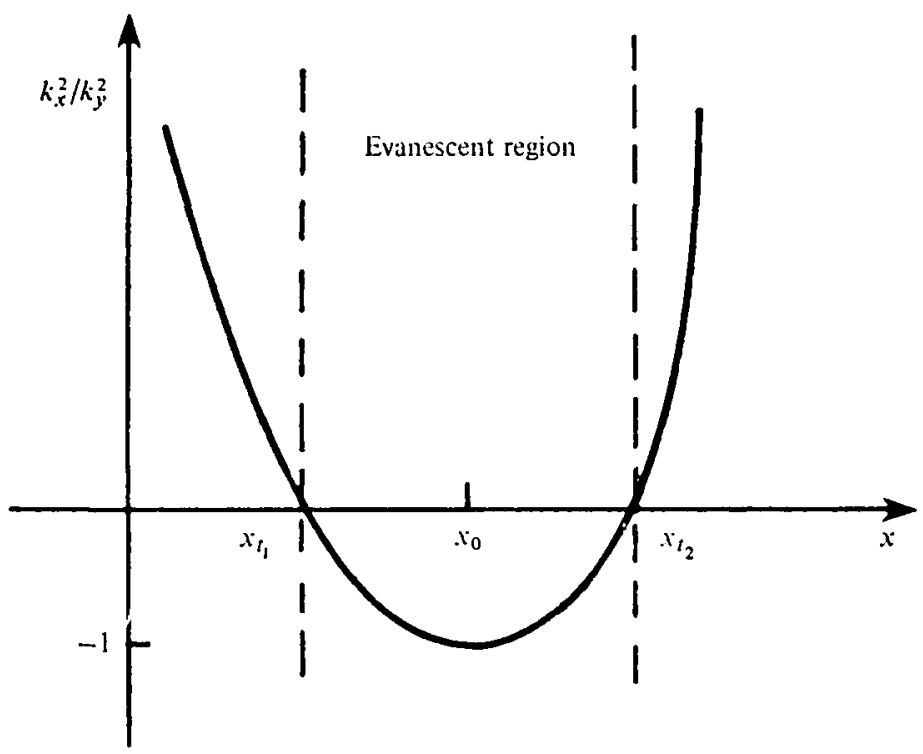

Fiaure 1. Plot of $k_{x}^{2} / k_{y}^{2}$ for hybrid waves when $k_{y} \gg k_{z}$.

A measure of the effect of magnetic shear on lower hybrid waves is the length over which $k_{x}$ grows from zero to $k_{y}$. Clearly, (11) indicates that this length is approximately $L$. Thus, the length $L$ is the effective shearing length for the parametric decay waves. A plot of $k_{x}^{2} / k_{y}^{2}$ as a function of $x$ illustrates in figure 1 the regions of propagation and evanescence of lower hybrid parametric decay-type waves.

At $x_{0}$ the wave reaches its rational surface $\left(k_{0}=0\right)$, and $k_{x}^{2}=-k_{y}^{2}$, so that $k_{1}^{2} /\left(k_{x}^{2}+k_{y}^{2}+k_{\|}^{2}\right)$ remains constant, the constant being imposed by the value of $\left(\omega^{2}-\omega_{L_{H}}^{2}\right) / \omega_{L_{H}}^{2}$. Since $x_{0}$ is the centre of the evanescent region, we conclude that magnetic shear determines locally the non-propagating portion of the $k$ spectrum. This is at variance with the effects of a density gradient. In the next section, we formulate the equation that describes the propagation of OTSI excited lower hybrid waves in a magnetically sheared plasma.

\section{Derivation of the OTSI equations}

We consider a dipole electric pump field $E_{0}(t)$ pointing in the $x$ direction

$$
\mathbf{E}_{0}(t)=\frac{1}{2} \hat{x}\left[E_{0}^{+} \exp \left(i \omega_{0} t\right)+E_{0}^{-} \exp \left(-i \omega_{0} t\right)\right],
$$

where the frequency $\omega_{0}$ is of the order of the lower hybrid frequency $\omega_{L H}$. Wave coupling is induced by the $\mathbf{E}_{0} \times \mathbf{B}_{0}$ electron drift velocity,

$$
\mathbf{v}_{D}=\frac{\left|\mathbf{E}_{0}\right|}{B_{0}}\left(-\hat{y}+\hat{z} \frac{x}{H}\right) \simeq-\hat{y} \frac{\left|\mathbf{E}_{0}\right|}{B_{0}}
$$

which is approximately constant since $x \ll H$. To describe lower hybrid waves we use a fluid model. The electrons are treated in the drift approximation, i.e. the electron equation of motion is

$$
\frac{\partial \mathbf{v}_{e}}{\partial t}=-\frac{e}{m_{e}} \mathbf{E}_{\mathrm{b}}+\frac{1}{B_{0}^{2}}\left(\frac{\partial \mathbf{E}}{\partial t} \times \mathbf{B}_{0}\right)-\frac{m_{e}}{e B_{0}^{2}} \frac{\partial^{2} \mathbf{E}_{\perp}}{\partial t^{2}}
$$


where the subscripts $\|$ and $\perp$ refer to directions parallel and perpendicular to $\mathbf{B}_{0}$. The ions are treated as unmagnetized, so that

$$
\frac{\partial \mathbf{v}_{i}}{\partial t}=\frac{e}{m_{i}} \mathbf{E}
$$

Since we are dealing with electrostatic waves, it is preferable to use the electric potential, i.e. $\mathbf{E}=-\nabla \phi$. Let

$$
n_{e}(\mathbf{r}, t)=n_{e}(\mathbf{r}) e^{i \omega t}+n_{e}^{*}(\mathbf{r}) e^{-i \omega t}
$$

be a low-frequency electron density fluctuation. The lower hybrid side-bands may be considered as the result of the nonlinear interaction between the pump and the low-frequency electron density fluctuations. In turn, electrons subjected to the fields of both the pump and its side-bands are influenced by a nonlinear ponderomotive potential $\phi_{p}$ which (completing the circle) drives the low-frequency electron density fluctuations. To describe these processes we employ the method used by Schmidt (1976). From (12)-(16) combined with Poisson's equation and the continuity equation, we obtain the nonlinear equation governing the electric potentials of the lower hybrid side-bands $\phi \pm$, oscillating at frequencies $\omega \pm \omega_{0}$,

$$
\left(\omega \pm \omega_{0}\right)^{2} \nabla^{2} \phi^{ \pm}-\omega_{L H}^{2}\left(\nabla^{2}+\frac{m_{i}}{m_{\epsilon}} \nabla_{\|}^{2}\right) \phi^{ \pm}= \pm \frac{i e \omega_{0}}{2 \epsilon_{0} B_{0}} \frac{E_{0}^{ \pm}}{1+\omega_{p_{e}}^{2} / \omega_{c_{\ell}}^{2}} \frac{\partial n_{e}}{\partial y}
$$

where $\epsilon_{0}$ is the vacuum dielectric constant.

We now introduce wave damping phenomenologically, replacing $\omega \pm \omega_{0}$ by $\omega+i \gamma_{L k} \pm \omega_{0}$, where $\gamma_{L k}$ is the natural lower hybrid wave damping rate. Since we are concerned with OTSI, we set $\omega=i \gamma$, where $\gamma$ is real. As a consequence of magnetic shear, $\nabla_{\mathrm{B}}^{2} \phi \pm$ is an explicit function of $x$, so that it is convenient to Fourier analyze (17) only in the $(y, z)$ plane. We shall use the notation

$$
\left.\begin{array}{l}
k^{2} \equiv k_{v}^{2}+k_{\varepsilon}^{2}, \\
\nabla^{2} \equiv \frac{d^{2}}{d x^{2}}-k^{2}, \\
\nabla_{\|}^{2} \equiv-k_{v}^{2}\left(\frac{x-x_{0}}{H}\right)^{2},
\end{array}\right\}
$$

so that (17) can now be written

$$
\begin{aligned}
\left\{\left\{\left[\omega_{0} \pm i\left(\gamma+\gamma_{L k}\right)\right]^{2}-\omega_{L H}^{2}\right\}\left(\frac{d^{2}}{d x^{2}}-k^{2}\right)+\omega_{L H}^{2} \frac{m_{i}}{m_{e}} k_{\nu}^{2}\left(\frac{x-x_{0}}{H}\right)^{2}\right\} & \\
= & = \pm \frac{e \omega_{0}}{\epsilon_{0} \omega_{p i}^{2}} \frac{\omega_{L H}^{2}}{2} \frac{E_{0}^{ \pm}}{B_{0}} k_{\nu} n_{e} .
\end{aligned}
$$

We now examine the low-frequency response.

As long as $k_{\|}^{2} m_{i} \gtrsim k^{2} m_{e}$, the electrons only respond parallel to the magnetic field lines and their motion is governed by

$$
\frac{\partial v_{e \|}}{\partial t}=-\frac{i k_{\|} e}{m_{e}}\left(\phi+\phi_{p}\right)+i k_{\|} V_{t e}^{2} \frac{\tilde{n}_{e}}{n_{0}} \simeq 0
$$


where we have taken into account the ponderomotive potential $\phi_{p}$ which drives the electron density fluctuations. It can be shown that in the limit of small growth rates, i.e. for $\left|\gamma / k_{\mathfrak{l}} V_{t i}\right|<1$, the ion density perturbation is

$$
n_{i} \simeq-\frac{\epsilon_{0}}{e} \frac{\phi}{\lambda_{D}^{2}} \frac{T_{e}}{T_{i}}
$$

where $\lambda_{D}=V_{t e} / \omega_{p e}$ is the Debye length. We assume that $\left(k^{2}-d^{2} / d x^{2}\right) \phi \ll \phi / \lambda_{D}^{2}$ so that Poisson's equation yields the quasi-neutrality condition $n_{i} \simeq n_{e}$. Using this condition, we obtain the expression for the electron density

$$
n_{e} \simeq \frac{\epsilon_{0}}{e} \frac{\phi_{p}}{\lambda_{D}^{2}\left(1+T_{i} / T_{e}\right)}
$$

The ponderomotive potential is defined by

$$
\phi_{p}=(\delta \mathbf{r} . \nabla) \phi
$$

where $\delta \mathbf{r}$ is the displacement of an electron about the oscillation centre. The displacement $\delta \mathbf{r}$ is produced by the pump field and results mainly from the $\mathbf{E}_{0} \times \mathbf{B}_{0}$ drift. Therefore,

$$
\delta \mathbf{r}=-\frac{i}{2} \frac{\hat{y}}{\omega_{0} B_{0}}\left(E_{0}^{+} \exp \left(i \omega_{0} t\right)-E_{0}^{-} \exp \left(-i \omega_{0} t\right)\right) .
$$

Substitution of $\delta \mathbf{r}$ given by (24) into (23), yields

$$
\phi_{p}=-\frac{k_{y}}{2 \omega_{0} B_{0}}\left(E_{0}^{+} \phi_{0}^{-}-E_{0}^{-} \phi^{+}\right) .
$$

When (19), (22) and (25) are combined, we obtain the coupled second-order differential equations that govern the potentials of the OTSI side-bands. Introducing the dimensionless quantities

$$
\left.\begin{array}{rl}
\Delta & \equiv \frac{\left(E_{0}^{+}\right)^{2} / B_{0}^{2}}{4 c_{s}^{2}\left(1+T_{i} / T_{e}\right)} \frac{\omega_{L H}^{2}}{\omega_{0}^{2}-\omega_{L H}^{2}}, \\
\bar{\gamma} & \equiv \frac{2\left(\gamma_{L k}+\gamma\right)}{\omega_{0}} \frac{\omega_{0}^{2}}{\omega_{0}^{2}-\omega_{L H}^{2}}
\end{array}\right\}
$$

and a new variable $\xi=x-x_{0}-L$, we can write the differential equations for OTSI potentials as

$$
\left.\begin{array}{l}
{\left[(1+i \bar{\gamma})\left(1-\frac{1}{k^{2}} \frac{d^{2}}{d \xi^{2}}\right)-\left(1+\frac{\xi}{L}\right)^{2}+|\Delta|\right] \phi^{+}=\Delta \phi^{-}} \\
{\left[(1-i \bar{\gamma})\left(1-\frac{1}{k^{2}} \frac{d^{2}}{d \xi^{2}}\right)-\left(1+\frac{\xi}{L}\right)^{2}+|\Delta|\right] \phi^{-}=\Delta^{*} \phi^{+}}
\end{array}\right\}
$$

In obtaining (27), we used the approximation $k_{y} \simeq k$, since $x_{0}$, defined in (9), must be located within the plasma. In the next section we investigate the solutions of (27). To simplify the notation, we shall assume that the phase of $E_{0}$ at $t=0$ is such that $E_{0}^{+}=E_{0}^{-}$so that $\Delta$ is real. 


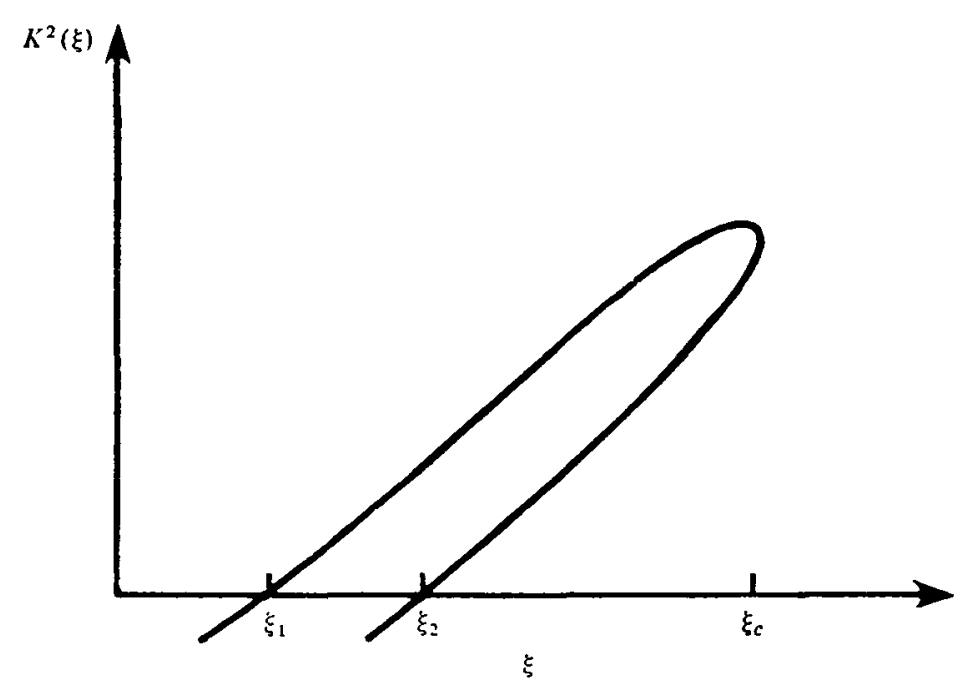

Figure 2. Lower hybrid wave propagation is allowed along the branches $\xi_{1} \leqslant \xi \leqslant \xi_{c}$ and $\xi_{2} \leqslant \xi \leqslant \xi_{c}$.

\section{Eigenvalue solutions}

The lowest-order WKB analysis of (27) gives an initial visualization of the wave behaviour under OTSI conditions. Assuming the potentials in (27) have the form $\phi^{ \pm} \propto \exp \left[i \int^{\xi} k_{x}\left(\xi^{\prime}\right) d \xi^{\prime}\right]$ we obtain the solutions

$$
K^{2}(\xi) \equiv k_{x}^{2}(\xi) / k^{2}=\left[(1+\xi / L)^{2}-1-\Delta-\bar{\gamma}^{2}\right] \pm\left\{\Delta^{2}\left(1+\bar{\gamma}^{2}\right)-\bar{\gamma}^{2}\left[(1+\xi / L)^{2}-\Delta\right]^{2}\right\}^{\frac{1}{2}} .
$$

$K^{2}(\xi)$ is real for a limited range of $\xi$. There are mirror points where $K^{2}(\xi)=0$, located at

$$
\xi_{1,2}=\frac{1}{2} L\left[\Delta \mp\left(\Delta^{2}-\bar{\gamma}^{2}\right)^{\frac{1}{2}}\right],
$$

and a conversion point, where the square root in (28) vanishes, located at

$$
\xi_{c}=L\left\{\left[\Delta+\frac{\Delta}{\bar{\gamma}}\left(1+\bar{\gamma}^{2}\right)^{\frac{1}{2}}\right]^{\frac{1}{2}}-1\right\} .
$$

Outside the region limited by these points $K(\xi)$ is complex. Retaining only wellbehaved solutions at $\xi \rightarrow \pm \infty$, we obtain spatially localized waves for real $\gamma \neq 0$. This confirms the absolute nature of the instability. The features of $K^{2}(\xi)$, given by (28), are summarized in figure 2 .

It is of interest to note that the region of wave propagation, i.e. the region where the solutions $K(\xi)$ are real, exists and has a finite extent only when $\Delta>\bar{\gamma}$. This region reduces to the point $\xi=\frac{1}{2} \Delta$ when $\Delta=\bar{\gamma}$ which is the condition for the threshold of the instability in the presence of a constant magnetic field $\mathbf{B}_{\mathbf{0}}$ (Kindel et al. 1972). Next we shall obtain a more complete solution of (27), first when $\Delta \gtrsim \bar{\gamma}$ and then when $\Delta \gg \bar{\gamma}$. 
For the case where the extent of the wave propagation region is small, i.e. when $\Delta \gtrsim \bar{\gamma}$, we can linearize (27), so that

$$
\left[(1 \pm i \bar{\gamma}) \frac{d^{2}}{d \eta^{2}}+\frac{2 \eta}{k L}-\Delta \mp i \bar{\gamma}\right] \phi^{ \pm}=-\Delta \phi^{\mp},
$$

where $\eta=k \xi$. Equation (31) is solved by a Fourier transform method (Drake et al. 1974) where the relation between any function $f(\eta)$ and its transform is given by

$$
f(\eta)=\int_{-\infty}^{+\infty} d \chi \exp (i \chi \eta) F(\chi)
$$

Taking the transform of (31), we can write (Liu \& Rosenbluth 1976)

$$
\phi \pm(\chi)=\psi^{ \pm} \exp \left\{-\frac{1}{2} i k L \int^{\chi} d \chi^{\prime}\left[\Delta \pm i \bar{\gamma}+\chi^{\prime 2}(1 \pm i \bar{\gamma})\right]\right\}
$$

where, after eliminating the $\psi^{-}(\chi)$ function, we obtain a second-order equation for $\psi^{+}(\chi)$ :

$$
\frac{d^{2} \psi^{+}}{d \chi^{2}}+\bar{\gamma} k L\left(1+\chi^{2}\right) \frac{d \psi^{+}}{d \chi}+\left(\frac{1}{2} k L\right)^{2} \Delta^{2} \psi^{+}=0
$$

By introducing $\Psi(\chi)$ defined by the equation

$$
\psi^{+}(\chi)=\psi(\chi) \exp \left[-\frac{1}{2} \bar{\gamma} k L \int^{\chi} d \chi^{\prime}\left(1+\chi^{\prime 2}\right)\right]
$$

one can eliminate the first-order derivative in (34) and obtain the Schrödinger equation

$$
d^{2} \tilde{\psi} / d \chi^{2}+A(\chi) \psi=0
$$

where

$$
A(\chi) \equiv\left(\frac{1}{2} k L\right)^{2}\left[\Delta^{2}-\bar{\gamma}^{2}\left(1+\chi^{2}\right)^{2}\right]-k L \bar{\gamma} \chi \text {. }
$$

In the propagation region $\chi$ is limited to values much less than one since it is assumed that $\bar{\gamma}$ is close to $\Delta$. In this limit $A(\chi)$ has two real roots, $\chi_{1}$ and $\chi_{\mathbf{2}}$ :

$$
\chi_{1,2}=-\frac{1}{k L \bar{\gamma}} \pm\left\{\left(\frac{1}{k L \bar{\gamma}}\right)^{2}+\frac{1}{2}\left(\frac{\Delta^{2}}{\bar{\gamma}^{2}}-1\right)\right\}^{\frac{1}{2}} \text {. }
$$

Thus well-behaved solutions of (36) exist if the eigenvalue equation

$$
\int_{\chi_{\mathrm{t}}}^{\chi_{\mathrm{a}}} A^{\frac{1}{2}}(\chi) d \chi=\left(n+\frac{1}{2}\right) \pi
$$

is satisfied. Let us define the variable $\tilde{\chi}=\chi+1 /(k L \bar{\gamma})$. Then $A(\chi)$ is zero at two symmetric values of $\tilde{\chi}$, namely at

$$
\tilde{\chi}_{1,2}=\mp\left\{\frac{1}{2}\left\{\frac{\Delta^{2}}{\bar{\gamma}^{2}}-1\right)+\frac{1}{k^{2} L^{2} \bar{\gamma}^{2}}\right\}^{\frac{1}{2}} .
$$

Therefore the integral in (40) may be written

$$
\frac{k L \bar{\gamma}^{(n)}}{2^{\frac{1}{2}}} \int_{-\tilde{\chi}^{2}}^{\tilde{\chi}_{2}}\left(\tilde{\chi}_{2}^{2}-\tilde{\chi}^{2}\right) d \tilde{\chi}=\left(n+\frac{1}{2}\right) \pi .
$$


In the limit where $L \rightarrow \infty, \tilde{\chi}_{2}$ must vanish in order to keep the integral in (41) finite. As a consequence one obtains the expected result for the shearless plasma, i.e. $\Delta=\bar{\gamma}$. The first-order corrections to this result, valid in the limit $\Delta k L \gg 1$, are determined from the solution of (41), which leads to the expression for the discrete eigenvalues

$$
\bar{\gamma}^{(n)}=\Delta-2 \frac{1}{2}(2 n+1) / k L \text {. }
$$

The second term, proportional to $1 / k L$, represents convective damping and is a measure of the difference between $\Delta$ and $\bar{\gamma}$, and thus a measure of the value of $k_{x} / k$ at $\xi_{c}$ as follows from (28). Similar results have been obtained for standing lower hybrid waves coupled to quasi-modes in a toroidal geometry (Porkolab 1977).

In the other limit, i.e. $\Delta \gg \bar{\gamma}$, the extent of the region of propagation is not limited to values of $\xi<L$, and we have to solve (27) in full. Below we summarize the analysis that yields the eigenvalue condition (Perkins \& Flick 1971), in the limit $\bar{\gamma} \ll \Delta \ll 1$.

Near the turning points $\xi_{1}$ and $\xi_{2}$ (where $\xi_{1,2} \ll L$ ) we linearize (27), i.e. we let $(1+\xi / L)^{2} \simeq 1+2 \xi / L$. It is of interest to notice that $\left(1 / k^{2}\right) d^{2} \phi / d \xi^{2}$ is of order $\Delta$, thus small compared with $\phi$. Rejecting terms of order $\bar{\gamma} \Delta$, we reduce (27) to

$$
\left(\frac{1}{k^{2}} \frac{d^{2}}{d \xi^{2}}+\frac{2 \xi}{L}-\Delta \mp i \bar{\gamma}\right) \phi^{ \pm}=-\Delta \phi \pm
$$

This set leads to two Airy equations:

$$
\left[\left(\frac{1}{k^{2}} \frac{d^{2}}{d \xi^{2}}+\frac{2 \xi}{L_{s}}-\Delta\right) \pm\left(\Delta^{2}-\bar{\gamma}^{2}\right)^{\frac{1}{2}}\right] \phi^{ \pm}=0
$$

The solutions of (44) are matched to the WKB solutions in the region $\xi \ll L_{s}$. Forbidding exponentially growing solutions for $\xi \rightarrow-\infty$, we obtain

$$
\phi=A \sin \left[\frac{\pi}{4}+\int_{\xi_{1}}^{\xi} K_{1}\left(\xi^{\prime}\right) d \xi^{\prime}\right]+B \sin \left[\frac{\pi}{4}+\int_{\xi_{2}}^{\xi} K_{2}\left(\xi^{\prime}\right) d \xi^{\prime}\right]
$$

asymptotically in the region $\xi>0$.

In the vicinity of the conversion point $\xi_{c}\left(\xi_{c} \gg L_{s}\right), d^{2} \phi / d \xi^{2} \gg k^{2} \phi$. We define

and (27) becomes

$$
(d \psi / d \xi)^{2}=k^{2}\left[(1+\xi / L)^{2}-\Delta\right]
$$

$$
\left[\frac{d^{2}}{d \xi^{2}}+\left(\frac{d \psi}{d \xi}\right)^{2} \mp i \bar{\gamma} \frac{d^{2}}{d \xi^{2}}\right] \phi \pm=-k^{2} \Delta \phi^{\mp}
$$

At lowest order, $d^{2} \phi^{ \pm} / d \xi^{2}=-(d \psi / d \xi)^{2} \phi^{ \pm}$. At next order,

$$
\left[\frac{d^{2}}{d \xi^{2}}+\left(\frac{d \psi}{d \xi}\right)^{2} \pm i \bar{\gamma}\left(\frac{d \psi}{d \xi}\right)^{2}\right] \phi \pm=-k^{2} \Delta \phi^{\mp} \text {. }
$$

This suggests that we look for solutions of the form

$$
\phi^{ \pm}=S \pm(\xi) \exp [i \psi(\xi)]
$$

Treating $(d \psi / d \xi)^{-1}$ as a smallness parameter and introducing (49) into (48), we obtain an equation for the leading terms:

$$
\frac{1}{k^{2}}\left[2 i \frac{d \psi}{d \xi} \frac{d}{d \xi} \mp i \bar{\gamma}\left(\frac{d \psi}{d \xi}\right)^{2}\right] S \pm=-\Delta S^{\mp}
$$


Since $(d \psi / d \xi)^{2} \simeq k^{2} \xi^{2} / L^{2}$ and since we confine our study to the range $\left(\xi-\xi_{c}\right) \ll \xi_{c}$, (50) leads to the Airy equation

$$
\left[\frac{1}{k^{2}} \frac{d^{2}}{d \xi^{2}}-\frac{\bar{\gamma}^{2} \xi_{c}\left(\xi-\xi_{c}\right)}{L^{2}}\right] S \pm=0
$$

The elimination of the non-physical exponentially growing component for $\xi>\xi_{c}$ leads to the solutions

$$
S \pm(\xi) \propto \sin \left\{\frac{\pi}{4}+k \int_{\xi_{c}}^{\xi} \bar{\gamma}\left[\frac{\xi_{c}\left(\xi_{c}-\xi^{\prime}\right)}{L^{2}}\right]^{\frac{1}{2}} d \xi^{\prime}\right\} .
$$

The integrand that appears on the right-hand side of (52) is the expansion of the argument of the square root that appears in the expression for $K^{2}(\xi)$, given by (28), when $\xi$ is near $\xi_{c}$ and $\xi_{c} \gg 1$. Substituting (52) into (49), one obtains

$$
\phi^{ \pm}=c\left\{\exp \left[R_{2}-\frac{1}{4} i \pi\right]+\exp \left[R_{1}+\frac{1}{4} i \pi\right]\right\}+D\left\{\exp \left[-R_{1}-\frac{1}{4} i \pi\right]+\exp \left[-R_{2}+\frac{1}{4} i \pi\right]\right\},
$$

where

$$
R_{1,2}=i k \int_{\xi_{c}}^{\xi} K_{1,2}\left(\xi^{\prime}\right) d \xi^{\prime}
$$

When the solutions obtained near $\xi_{1,2}$ (i.e. (45)) and near $\xi_{c}$ (i.e. (49)) are matched to the WKB solutions in the centre of the region of propagation, we obtain the eigenvalue equation

$$
I \equiv k \int_{\xi_{1}}^{\xi_{c}} K_{1}(\xi) d \xi-k \int_{\xi_{1}}^{\xi_{c}} K_{2}(\xi) d \xi=\left(n+\frac{1}{2}\right) \pi
$$

where $n$ is an integer indicating the number of nodes in the propagation region.

Under the assumption $1 \gg \Delta \gg \bar{\gamma}$, the expression for $K^{2}(\xi)$ in (28) can be written

$$
K_{1,2}\left(\xi^{\prime}\right)=\left[\xi^{\prime}-\Delta \pm\left(\Delta^{2}-\bar{\gamma}^{2} \xi^{\prime 2}\right)^{\frac{1}{1}}\right]^{\frac{1}{2}}
$$

where $\xi^{\prime}=(1-\xi / L)^{2}-1$. In terms of $\xi^{\prime}$ the region of propagation is defined by $\xi_{1}^{\prime}=0, \xi_{2}^{\prime}=2 \Delta$ and $\xi_{c}^{\prime}=\Delta / \bar{\gamma}$. The integral $I$ may be separated into three parts, i.e. $I=I_{1}+I_{2}+I_{3}$ where

$$
\left.\begin{array}{l}
I_{1} \equiv k \int_{\xi_{1}^{\prime}}^{\xi_{2}^{\prime}} K_{1}\left(\xi^{\prime}\right) d \xi^{\prime} \simeq \frac{1}{2} k L \int_{0}^{2 \Delta}\left(\xi^{\prime}\right)^{\frac{1}{2}} d \xi^{\prime}, \\
I_{2} \equiv k \int_{\xi_{2}^{\prime}}^{2^{+}-1}\left[\frac{K_{1}^{2}\left(\xi^{\prime}\right)-K_{2}^{2}\left(\xi^{\prime}\right)}{K_{1}\left(\xi^{\prime}\right)+K_{2}\left(\xi^{\prime}\right)}\right] d \xi^{\prime} \simeq \frac{1}{2} \Delta k L \int_{2 \Delta}^{1} \frac{d \xi^{\prime}}{\left(\xi^{\prime}\right)^{\frac{1}{2}}}, \\
I_{3} \equiv k \int_{2^{1}-1}^{\xi_{c}^{\prime}}\left[\frac{K_{1}^{2}\left(\xi^{\prime}\right)-K_{2}^{2}\left(\xi^{\prime}\right)}{K_{1}\left(\xi^{\prime}\right)+K_{2}\left(\xi^{\prime}\right)}\right] d \xi^{\prime} \simeq \frac{1}{2} \bar{\gamma} k L \int_{1}^{\xi_{c}^{\prime}\left(\xi_{c}^{\prime 2}-\xi^{\prime 2}\right)^{\frac{1}{2}}} d \xi^{\prime} \\
\xi^{\prime}
\end{array}\right\}
$$

where we have made the following approximations: for $\xi^{\prime}<1$, we neglected $\xi^{\prime}$ compared to 1 ; for $\xi^{\prime}>1$ we neglected 1 compared to $\xi^{\prime}$ : and for $\xi^{\prime}>2 \Delta$ we neglected $\Delta$ compared to $\xi^{\prime}$. The eigenvalue equation then reduces to

$$
1-\frac{2}{3}(2 \Delta)^{\frac{1}{2}}+\ln \left(\frac{2 \Delta}{\bar{\gamma}^{(n)}}\right)=\frac{(2 n+1) \pi}{\Delta k L}
$$


where $\bar{\gamma}^{(n)}$ is the $n$th eigenvalue. When $\Delta / \bar{\gamma}$ is sufficiently large compared to 1 , the logarithmic term dominates on the left-hand side of (57). In this limit the eigenvalues

$$
\bar{\gamma}^{(n)}=2 \Delta \exp \left[-\frac{(2 n+1) \pi}{\Delta k L}\right]
$$

have an accumulation point at $\bar{\gamma}=0$ (that is for $\gamma_{g}=-\gamma_{L k}$ ) when $n \rightarrow \infty$. However, there is only a trend toward accumulation since the accumulation point occurs outside the plasma where $\xi_{c} / L \rightarrow \infty$.

\section{Discussion}

We recall that our results have been obtained under the assumption of small growth rates, i.e. $\gamma^{(n)}<k_{0} V_{t i}$, in order to be able to describe threshold. This condition is most stringent for the fundamental mode, i.e. for $n=0$. Also, it is violated at the mode rational surface, where $k_{\|}=0$. However, we only need this condition to hold in the propagation region, so that the real condition of validity is $\gamma^{(0)}<k_{\|}\left(\xi_{i}\right) V_{t i}$. In order to check the results for $\bar{\gamma}^{(n)}$ given by (42) and (58), we evaluated numerically the integral in (54). Let $\alpha=\bar{\gamma} / \Delta$ so that the points limiting propagation region can be written

$$
\left.\begin{array}{rl}
\xi_{1,2} & \simeq \frac{1}{2} \Delta L\left[1 \mp\left(1-\alpha^{2}\right)^{\frac{1}{2}}\right] \ll 1, \\
\xi_{c} & \simeq L_{s}(1-\alpha) /\left(\alpha+\alpha^{\frac{1}{2}}\right) .
\end{array}\right\}
$$

In this case the integral in (54) is independent of $\Delta$ and may be expressed in the form

$$
\int_{0}^{\frac{1-\alpha}{\alpha+\alpha^{\frac{1}{7}}}}\left(\frac{1-\alpha^{2}-4 \alpha^{2} y}{2 y}\right)^{\frac{1}{2}} d y=\frac{\left(n+\frac{1}{2}\right) \pi}{\Delta k L} .
$$

We have verified that the analytic results obtained above are recovered in both limits, i.e. $\Delta \gtrsim \bar{\gamma}$ and $\Delta \gg \bar{\gamma}$. The results are presented in figure 3 .

The linear dependence of $\bar{\gamma} / \Delta$ on $\pi(2 \Delta k L)^{-1}$ for $\Delta \gtrsim \bar{\gamma}^{(n)}$ is in agreement with the result given by (42) until $\bar{\gamma}^{(n)} \simeq 0.7 \Delta$. The dotted straight line in figure 3 represents the analytical result of (42). For $\bar{\gamma}^{(n)}<0 \cdot 25$, we recover the exponential result within an additive constant. Indeed, for these values of $\bar{\gamma}<0.25 \Delta$ and $\Delta<1$ the numerical result is

$$
\log \left(\Delta / \bar{\gamma}^{(n)}\right)=\frac{(2 n+1) \pi}{\Delta k L}-1 \cdot 08
$$

compared with the result obtained from (53)

$$
\log \left(\Delta / \bar{\gamma}^{(n)}\right)=\frac{(2 n+1) \pi}{\Delta k L}-1 \cdot 69
$$

We note that, in the case where $\gamma_{L}$ is zero, the instability is not completely eliminated by shear. This is in contrast to those cases where there is a linear reduction of growth rates proportional to $(\Delta k L)^{-1}$ and the instability is suppressed (Perkins \& Flick 1971; Liu \& Rosenbluth 1976). The slower reduction 


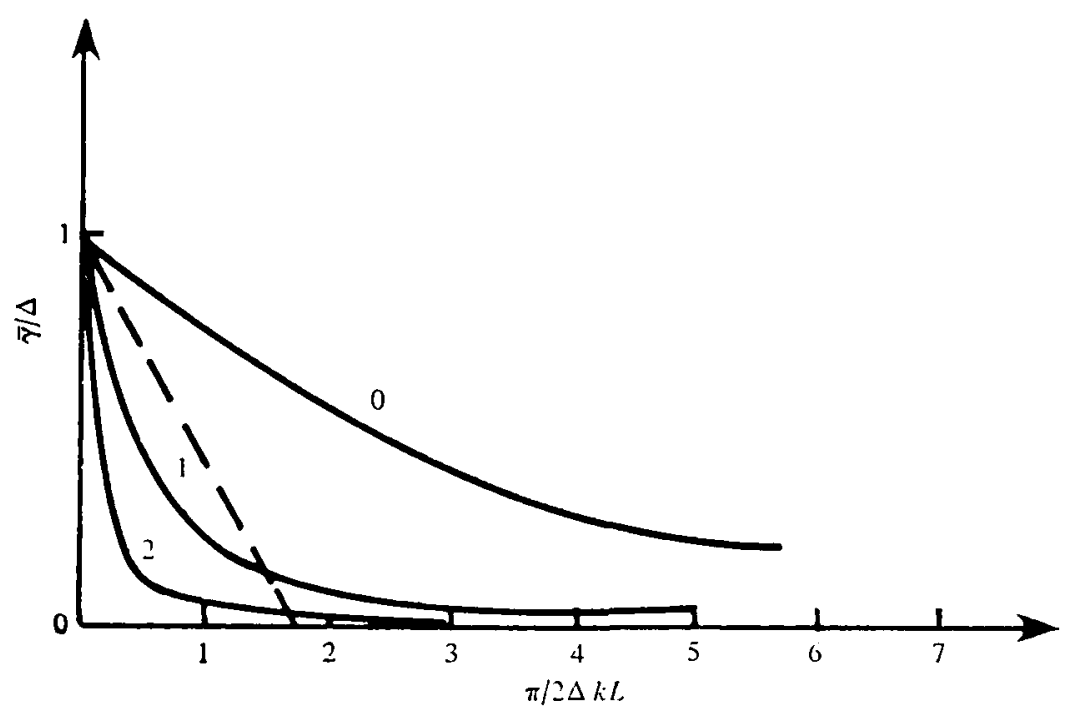

FIGURE 3. Eigenvalues $\bar{\gamma}^{(n)} / \Delta$ in the limit $\Delta, \bar{\gamma}^{(n)} \ll 1$. The dotted straight line indicates the result of (42).

of growth rates, compared with linear reduction of $(\Delta k L)^{-\mathbf{1}}$, is related to the behaviour of the group velocity of lower hybrid waves. It has been pointed out that convective damping arises owing to loss of energy from the strong coupling region (Perkins \& Flick 1971). This loss is in the direction of the inhomogeneity, i.e. in the $x$ direction for the sheared plasma studied here. The strong coupling region is defined as the length over which the total wave vector rotates by $\frac{1}{4} \pi$. This definition of the strong coupling region is valid as long as the group velocity is constant or increases. However, lower hybrid waves have a group velocity

$$
v_{a_{x}}=\frac{\omega_{L H}^{2}-\omega_{0}^{2}}{\omega_{0}} \frac{k_{x}(x)}{k^{2}+k_{x}^{2}(x)}
$$

which decreases when $k_{x}>k$, and energy loss along $x$ is reduced as $k$ increases. We believe that this mechanism is responsible for the exponential reduction of growth rates.

The major part of this research was done at the Centre de Recherche en Physique des Plasmas of the Ecole Polytechnique de Lausanne and was supported by Swiss Fonds National. This work was completed when one of the authors (J.M.W.) was at the Laboratory of Plasma Studies, Cornell University, supported by the United States Department of Energy Contract, EY-76S-023170 and when another of the authors (A.H.K.) was at the Princeton Plasma Physics Laboratory, supported by the United States Department of Energy Contract EY-76C-02-3073. 


\section{REFERENCES}

Berger, R. L. \& Perkins, F. W. 1976 Phys. Fluids, 19, 406.

Berger, R. L., Chen, L., Kaw, P. K. \& Perkins, F. W. 1977 Phys. Fluids, 20, 1864.

Chang, R. R. H. \& Porgolab, M. 1973 Phys. Rev. Lett. 31, 1241.

Chang, R. P. H., \& Porkolab, M. 1974 Phys. Rev. Lett. 32, 1227.

Chen, L. \& Berger, R. L. 1977 Nucl. Fusion, 17, 779.

Chu, T. K., Bernabei, S. \& Motley, R. W. 1973 Phys. Rev. Lett. 31, 211.

Frmed, B. D., Ikemura, T., Nishikawa, K. \& Schmidt, G. 1976 Phys. Fluids, 19, 1975.

Hooke, W. M. \& Bernabet, S. 1972 Phys. Rev. Lett. 29, 1218.

Kindel, J. M., OkUda, H. \& Dawson, J. M. 1972 Phys. Rev. Lett. 29, 995.

KUo, Y. Y. \& ChEN, L. 1976 Phys. Fluids, 19, 1223.

Lid, C. S. \& Rosengluth, M. N. 1976 Phys. Fluids, 19, 967.

Oтт, E. 1975 Phys. Fluids, 18, 566.

Perkins, F. W. \& Futck, J. 1971 Phys. Fluids, 14, 2012.

Porkolab, M. 1974a Phys. Fluids, 17, 1432.

Porkoras, M. $1974 b$ Proceedings of the Symposium on Plasma Heating in Toroidal Devices, Bologna, Italy, p. 28.

Porkolab, M. 1977 Phys. Fluids, 20, 2058.

Rogister, A. \& Hasselberg, G. 1976 Phys. Fluids, 19, 108.

Rosenbluth, M. N. 1972 Phys. Rev. Lett. 29, 565.

SCHMrDT, G. 1976 3rd International Meeting on Theoretical and Experimental Aspects of Heating in Toroidal Plasmas, Grenoble, France, vol. 2, p. 15.

Strx, T. H. 1965 Phys. Rev. Lett. 15, 878.

Wersinger, J. M., Kritz, A. H. \& Troyon, F. 1976 3rd International Meeting on Theo. retical and Experimental Aspects of Heating in Toroidal Plasmas, Grenoble, France, vol. 1 , p. 169 . 